\title{
IL-1 receptor antagonist therapy mitigates placental dysfunction and perinatal injury following Zika virus infection
}

\author{
Jun Lei, ${ }^{1}$ Meghan S. Vermillion, ${ }^{2,3}$ Bei Jia, ${ }^{1}$ Han Xie, ${ }^{1}$ Li Xie, ${ }^{1}$ Michael W. McLane, ${ }^{1}$ \\ Jeanne S. Sheffield, ${ }^{1}$ Andrew Pekosz, ${ }^{2}$ Amanda Brown, ${ }^{4}$ Sabra L. Klein, ${ }^{2,5}$ and Irina Burd ${ }^{1,4}$ \\ IIntegrated Research Center for Fetal Medicine, Department of Gynecology and Obstetrics, Johns Hopkins University \\ School of Medicine, Baltimore, Maryland, USA. ${ }^{2}$ W. Harry Feinstone Department of Molecular Microbiology and \\ Immunology, Johns Hopkins Bloomberg School of Public Health, Baltimore, Maryland, USA. ${ }^{3}$ Department of Molecular \\ and Comparative Pathobiology, ${ }^{4}$ Department of Neurology, Johns Hopkins University School of Medicine, Baltimore, \\ Maryland, USA. ${ }^{5}$ Department of Biochemistry and Molecular Biology, Johns Hopkins Bloomberg School of Public Health, \\ Baltimore, Maryland, USA.
}

Zika virus (ZIKV) infection during pregnancy causes significant adverse sequelae in the developing fetus, and results in long-term structural and neurologic defects. Most preventive and therapeutic efforts have focused on the development of vaccines, antivirals, and antibodies. The placental immunologic response to ZIKV, however, has been largely overlooked as a target for therapeutic intervention. The placental inflammatory response, specifically IL-1 $\beta$ secretion and signaling, is induced by ZIKV infection and represents an environmental factor that is known to increase the risk of perinatal developmental abnormalities. We show in a mouse model that maternally administrated IL-1 receptor antagonist (IRA; Kineret, or anakinra), following ZIKV exposure, can preserve placental function (by improving trophoblast invasion and placental vasculature), increase fetal viability, and reduce neurobehavioral deficits in the offspring. We further demonstrate that while ZIKV RNA is highly detectable in placentas, it is not correlated with fetal viability. Beyond its effects in the placenta, we show that IL-1 blockade may also directly decrease fetal neuroinflammation by mitigating fetal microglial activation in a dose-dependent manner. Our studies distinguish the role of placental inflammation during ZIKV-infected pregnancies, and demonstrate that maternal IRA may attenuate fetal neuroinflammation and improve perinatal outcomes.

\section{Authorship note: JL and MSV contributed equally to this work.}

Conflict of interest: The authors have declared that no conflict of interest exists.

Copyright: $\odot 2019$ American Society for Clinical Investigation

Submitted: June 4, 2018 Accepted: February 14, 2019 Published: February 28, 2019

Reference information: $\mathrm{JCl}$ Insight. 2019;4(7):e122678. https://doi.org/10.1172/jci. insight.122678.

\section{Introduction}

Zika virus (ZIKV) outbreaks have been reported in more than 50 countries and territories since 2015 (1), with more than 3,000 confirmed infections in fetuses (2). ZIKV, a positive-stranded RNA virus, can transmit via mosquito vectors, sexual contact, and blood transfusion (3-5). The virus typically causes asymptomatic infections in adults, but can cause severe fetal sequelae when infection occurs during pregnancy. ZIKV infection of pregnant women is associated with fetal abnormalities - collectively referred to as the congenital Zika syndrome (6) - that are characterized by a spectrum of developmental defects in the human fetus, including microcephaly, decreased brain tissue and structural abnormalities, limb contractures, as well as neurological sequelae. While much of the attention has been focused on the biology of the ZIKV and its mosquito vector, the immunologic complexity of pregnancy and placental immune responses to ZIKV have been largely ignored. Currently, there is no specific treatment for ZIKV infection. Although there are more than 50 candidate ZIKV vaccines and antibodies being developed (7-9), their use in pregnant women is still pending completion of clinical trials and FDA approval. Moreover, the preventive and therapeutic strategies are virus specific, and targeted toward the virus itself, and in some cases still do not mitigate adverse perinatal outcomes (10). Although ZIKV infection can have direct effects on fetal tissues, there is evidence that the long-term perinatal and neurologic sequelae are associated with increased inflammation and dysregulation of the placenta (11-13). 
Studies in vitro have shown that ZIKV directly infects endothelial cells, placental trophoblast cells, Sertoli cells, glomerular cells, neurons, and fetal microglia (14-18).Increased secretion of proinflammatory cytokines, including IL-1 $\alpha$ and IL-1 $\beta$, from ZIKV-infected cells might contribute to cell death (19, 20). Furthermore, $\mathrm{IL}-1 \beta$ is elevated in the serum of nonpregnant ZIKV-infected patients $(7,21)$. Whether IL-1 $\beta$ is upregulated in the placenta following ZIKV infection remains unknown and formed the basis of the current study.

IL-1 receptor antagonist (IRA) is an endogenous molecule present in amniotic fluid and cord blood, and circulates systemically at higher levels in pregnant compared with nonpregnant women (22). Animal studies demonstrate that treatment during pregnancy with exogenous IRA (Kineret, or anakinra, a class B drug in pregnancy) can protect against placental and neurodevelopmental defects induced by in utero inflammation (23-25). These observations provided the foundation for our investigation of the role of IL-1 signaling in maternal ZIKV-induced fetal brain injury and its potential as a therapeutic target to prevent or attenuate congenital Zika syndrome. We hypothesized that therapeutic agents that target inflammation, e.g., through antagonism of IL-1 signaling, could mitigate placental inflammation and long-term adverse perinatal outcomes.

In this study, we used an immunocompetent mouse model of intrauterine ZIKV infection (13 ) to explore the effects of placental ZIKV infection on trophoblast invasion and placental inflammation, including IL-1 $\beta$ production. We (13) and others (26-30) have demonstrated that systemic inoculation of virus (i.e., intraperitoneal/subcutaneous) does not result in transplacental (i.e., vertical) transfer of ZIKV in immunocompetent mice, in part because ZIKV does not antagonize the IFN response in mice as it does in humans (26). To circumvent this problem in mice, several models have been developed to study ZIKV pathogenesis during pregnancy in mice, including deletion or depletion of type I IFNs (28, 31-33), development of humanized STAT2 mice (26), and direct inoculation into the female reproductive tract $(13,34-36)$. In the present study, immunocompetent dams were infected in utero with ZIKV to systematically evaluate the impact of ZIKV on the placental inflammatory responses and test the efficacy of maternally administered IRA in reducing placental inflammation, placental dysregulation, and the subsequent adverse neonatal outcomes. Finally, we tested the direct effects of exogenous IRA on perinatal microglial infection and proliferation in vitro. Collectively, we show that IRA treatment inhibits microglial activation in the perinatal brain both directly and indirectly through preservation of trophoblast invasion and vascular remodeling in the placenta.

\section{Results}

In utero exposure to ZIKV causes placental dysfunction and developmental abnormalities in offspring. Consistent with previous studies (13), intrauterine ZIKV inoculation of immunocompetent pregnant mice at E10 (i.e., during the critical period of placental completion; ref. 37) caused detectable placental infection 48 hours after inoculation (48 hpi) that was similar across distinct strains of ZIKV (Figure 1A). Following ZIKV infection, there was a significant reduction in trophoblast invasion into the mesometrial triangle $(P$ $<0.05,1$-way ANOVA, Figure 1, B and C) and decreased vimentin expression in placental villi $(P<0.05$, 1-way ANOVA, Figure 1, D and E) compared with placentas from mock-inoculated dams.

Infection with diverse strains of ZIKV significantly reduced fetal viability 48 hpi $(P<0.05,1$-way ANOVA, Figure 1F); placental ZIKV RNA copies, however, did not correlate with fetal survival $\left(r^{2}\right.$ $=0.3034, P=0.309$, Figure $1 \mathrm{G}$ ). A subset of dams was allowed to carry to term, and developmental abnormalities were quantified in pups through postnatal day P8. The pups exposed in utero to ZIKV at E10 exhibited abnormal development, including limb contractures (Figure $1 \mathrm{H}$ ), congenital syndactyly (Figure 1I), and kinked tails (Figure 1J). Collectively, the occurrence of these developmental abnormalities in ZIKV-exposed pups was calculated to be $1.8 \%$ (Table 1). These data suggest an association between placental dysfunction and adverse perinatal outcomes following in utero ZIKV exposure, regardless of placental viral load.

In utero exposure to ZIKV increases placental IL-1 $\beta$. Because ZIKV infection induces peripheral IL-1 $\beta$ secretion in nonpregnant ZIKV-infected humans and mice (38), we sought to determine whether ZIKV infection during pregnancy could similarly upregulate IL-1 $\beta$ as well as other proinflammatory mediators in the placenta. Using multiplexed arrays, we measured the mRNA expression of 96 immune-related genes and observed that $I l 1 \beta$ mRNA expression, but not expression of other proinflammatory cytokines, was upregulated in the placenta 6 hours after in utero ZIKV inoculation $(P<0.05$, Student's $t$ test, Figure 2A and Supplemental Table 1; supplemental material available online with this article; https:// 
A

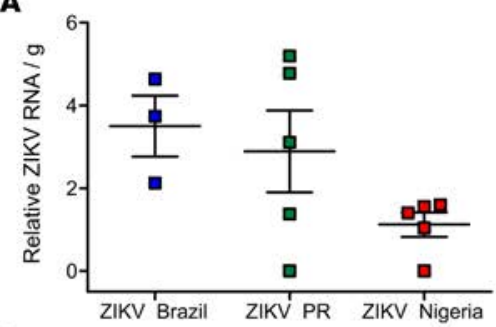

B

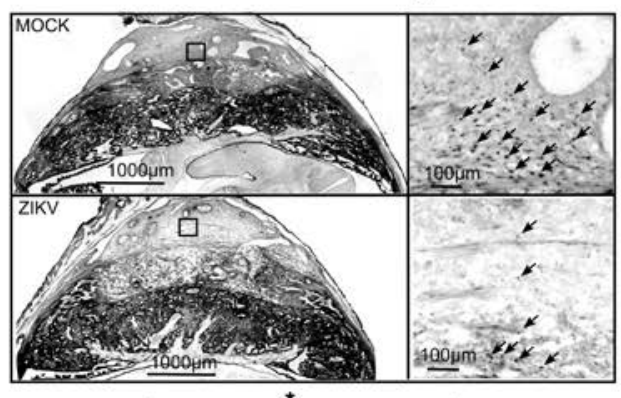

C

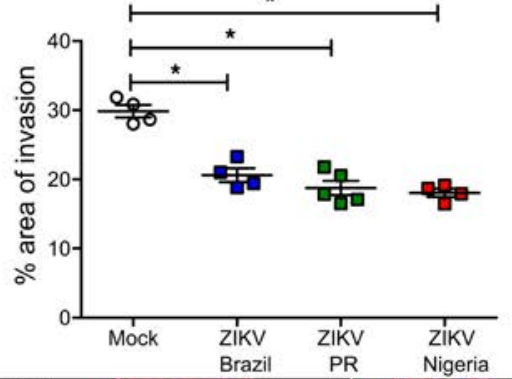

D

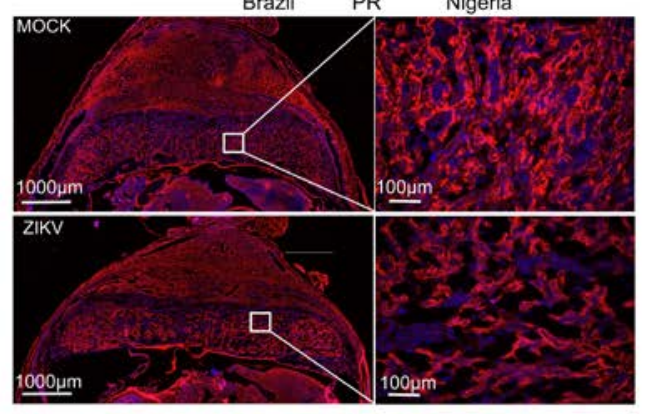

E

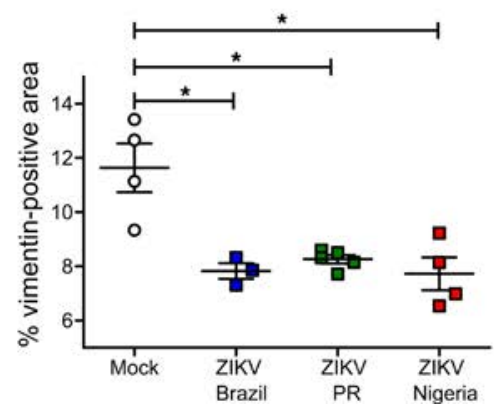

$\mathbf{F}$

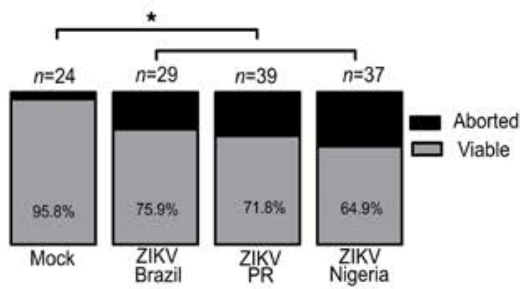

G

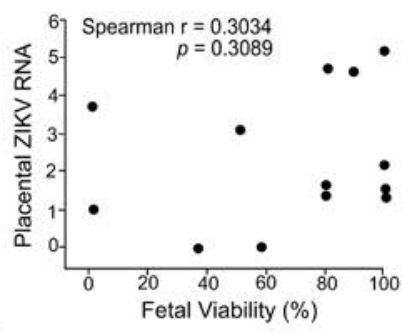

H

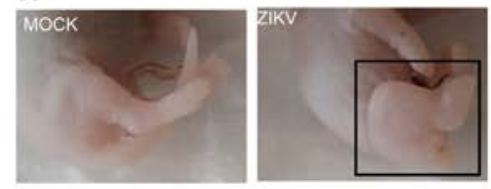

I

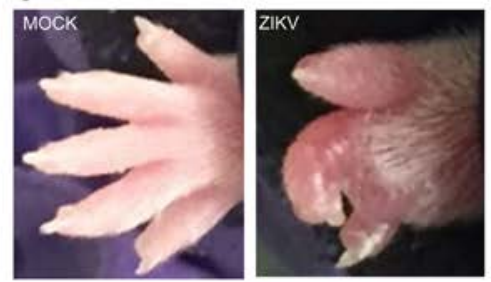

J

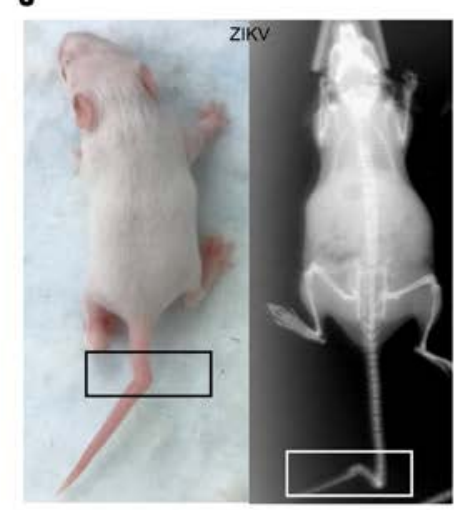

Figure 1. Historic and contemporary strains of ZIKV infect the placenta and cause placental dysfunction and developmental abnormalities in offspring. At E10, pregnant CD-1 mice received an intrauterine injection of $10^{6} \mathrm{TCID}_{50}$ units of the 2015 Brazil, 2015 Puerto Rico (PR), or 1968 Nigeria ZIKV, or vehicle (mock). (A) ZIKV RNA was isolated and quantified by qRT-PCR from placentas collected 48 hours after inocuIation (48 hpi). (B) Representative fluorescence immunostaining for cytokeratin (black puncta, arrows) to label trophoblast cells in mock- or ZIKV-infected (Brazil strain) placentas. Right: High magnifications of black boxes (located in the mesometrial triangle) in the left panels. Scale bars: $1000 \mu \mathrm{m}$ (left), $100 \mu \mathrm{m}$ (right). (C) Quantitative analysis of trophoblast density (as a measure of trophoblast invasion) in the mesometrial triangle. (D) Representative fluorescence immunostaining for vimentin (red) to label endothelial cells in mock- or ZIKV-infected (Brazil strain) placentas. The right panels are high magnifications of the white boxes (located in the villi) in the left panels. Scale bars: $1000 \mu \mathrm{m}$ (left), $100 \mu \mathrm{m}$ (right). (E) Quantitative analysis of endothelial cell density in the placental villi. (F) Fetal viability quantified as the percentage of viable fetuses within the inoculated uterine horn ( $n=$ total number of fetuses from 4 to 5 dams per group). (C) Correlation between ZIKV RNA in placentas and fetal viability. (H-J) Representative images of limb contracture (arthrogryposis) (H), fused digits (syndactyly) (I), and kinked tails (J, left panel, gross image; right panel, radiograph) in pups born to ZIKV-infected dams. $\chi^{2}$ test $(\mathbf{F})$, Spearman's correlation analysis (G), and 1-way ANOVA with Bonferroni's multiple comparisons test $(\mathbf{A}, \mathbf{C}$, and $\mathbf{E}) .{ }^{*} P<0.05$. 
Table 1. Frequency of congenital malformations

\begin{tabular}{lcc}
\hline & ZlkV-infected & Mock-infected \\
Total pups $(n)$ & 322 & 258 \\
Limb and tail malformation $(n)$ & 6 & 0 \\
Frequency $(\%)$ & 1.8 & 0
\end{tabular}

doi.org/10.1172/jci.insight.122678DS1 ). At 6 hpi, the concentration of IL-1 $\beta$ was also significantly increased in ZIKV-infected placentas as compared with placentas from mock-inoculated dams $(P<0.05$, Student's $t$ test, Figure 2B). The induction of IL-1 $\beta$ in placenta was transient, as these changes were less pronounced at 48 hpi (Supplemental Table 2 and Supplemental Figure 1A). Peripherally, in the maternal spleen, there was no increase in the concentrations of IL-1 $\beta$ at 6 hours following intrauterine ZIKV inoculation (Figure 2B), suggesting that in this model, IL-1 $\beta$-mediated inflammation was occurring locally in the placenta. To determine whether the ZIKV-induced increase in placental IL-1 $\beta$ required replicating virus, dams were inoculated with inactivated ZIKV and placentas were collected 6 hpi. Live, but not inactivated, ZIKV caused an increase in placental IL-1 $\beta$ concentrations (Supplemental Figure 1B), illustrating that virus replication is necessary to induce IL-1 $\beta$ in the placenta.

To examine whether the placental dysregulation (Figure 1, B-E) was caused by the upregulation of IL-1 $\beta$ (Figure 2B), we treated dams with IRA 3 hours after mock or ZIKV infection. Blocking IL-1 receptor signaling with IRA increased ZIKV copies in the placenta $(P<0.05$, Student's $t$ test, Figure 2C), but not in the maternal spleen or fetal head (Figure 2C). Consistent with previous results (Figure $1 \mathrm{~F}$ ), fetal viability was significantly reduced in ZIKV- compared with mock-inoculated dams 48 hpi $(P<0.05$, $\chi^{2}$, Figure 2D); in contrast, the viability of fetuses from IRA-treated ZIKV-infected dams significantly improved as compared with ZIKV-infected dams $\left(P<0.05, \chi^{2}\right.$, Figure 2D). The ZIKV-induced reduction in fetal viability was reversed by coadministration of IRA not only at 3 hpi, but at 1 or 24 hpi, suggesting that there is an extended window of opportunity for treatment (Supplemental Figure 1C).

Maternal IRA administration preserves placental development during intrauterine ZIKV infection. IHC of placental trophoblasts (cytokeratin) and endothelial cells (vimentin) was utilized to assess the effect of IRA treatment on placental development. Compared with PBS-treated, ZIKV-infected dams, IRA administration during ZIKV infection significantly improved the migration of trophoblasts into the mesometrial triangle $(P<0.05,1$-way ANOVA, Figure 3, A and B). There were abundant trophoblast cells around or at the decidual spiral arteries in placentas from mock-infected dams (Supplemental Figure 2, white arrows) $(39,40)$. ZIKV infection reduced the appearance of trophoblasts in the spiral arteries, whereas treatment of ZIKV-infected dams with IRA increased the amount of trophoblast cell invasion into the decidual spiral arteries (Supplemental Figure 2). The density of trophoblasts within the placental labyrinth was also markedly increased following IRA treatment of ZIKV-infected dams $(P<0.05,1$-way ANOVA, Figure 3, $A$ and B). Similarly, placental vascularity, as measured by the density of vimentin staining, was increased by IRA administration to ZIKV-infected dams $(P<0.05,1$-way ANOVA, Figure 3, D and E). Taken together, these data illustrate that by reducing placental inflammation with IRA, ZIKV-induced placental dysfunction is mitigated despite the presence of ZIKV RNA (Figure 2C).

Maternal IRA administration mitigates the behavioral consequences of ZIKV and inhibits microglial expression in neonatal brains. To determine the effects of IRA on restoring neurocognitive function in neonates born to ZIKV-infected dams, neurological behavioral tests were performed at P5 and P9, as previously described (41). At P5, pups born to ZIKV-infected dams took significantly longer to complete tasks, including negative geotaxis, cliff aversion, and surface righting, as compared with pups from mock-inoculated dams $(P<0.05,1$-way ANOVA, Figure 4A). IRA administration to ZIKV-infected dams reversed the behavioral abnormalities in pups and significantly shortened the performance times of these tests $(P$ $<0.05$, 1-way ANOVA, Figure 4A), as compared with pups exposed to ZIKV without IRA treatment.

Neuroinflammation can contribute to neurocognitive deficits in neonatal mice (42-44). IHC for Iba-1, a marker of microglia, was used to assess neuroinflammation in the brains of pups at P5. Consistent with previous reports (13), Iba-1 expression was significantly greater in the brains of pups born to ZIKV-infected dams ( $P<0.05,1$-way ANOVA, Figure $4, \mathrm{~B}$ and $\mathrm{C}$ ), and IRA administration significantly decreased the expression of Iba-1 to levels comparable to brains from pups born 
A

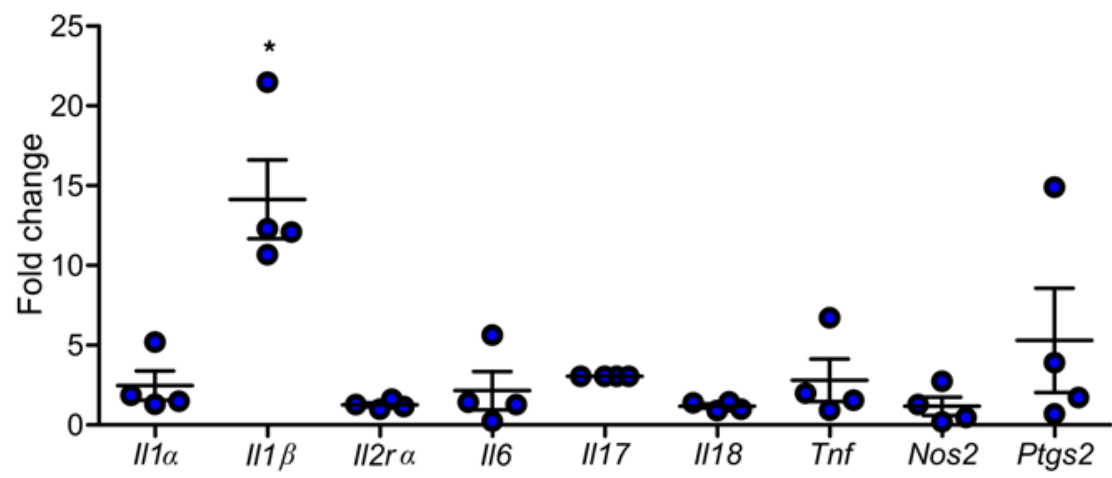

B

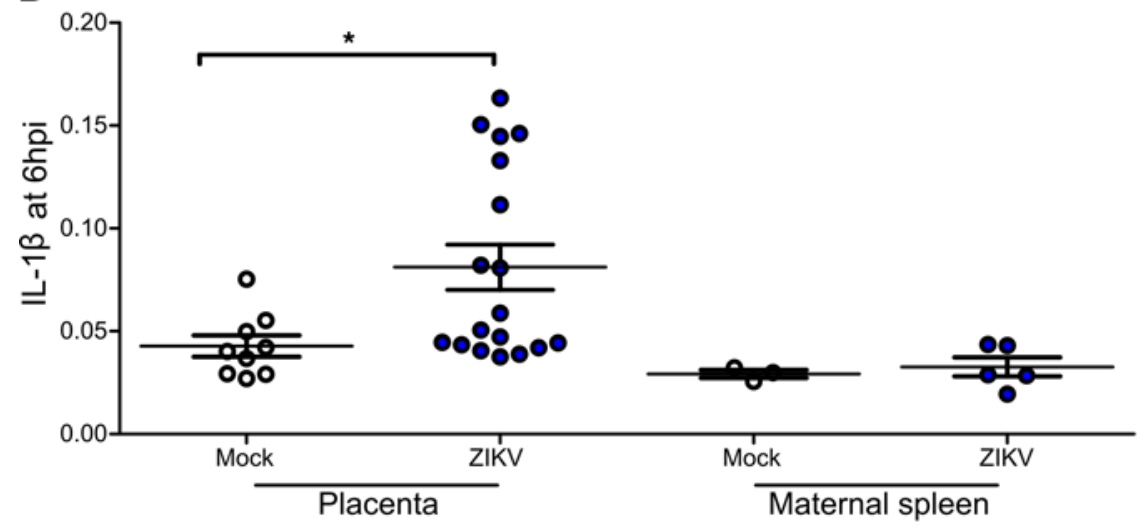

C

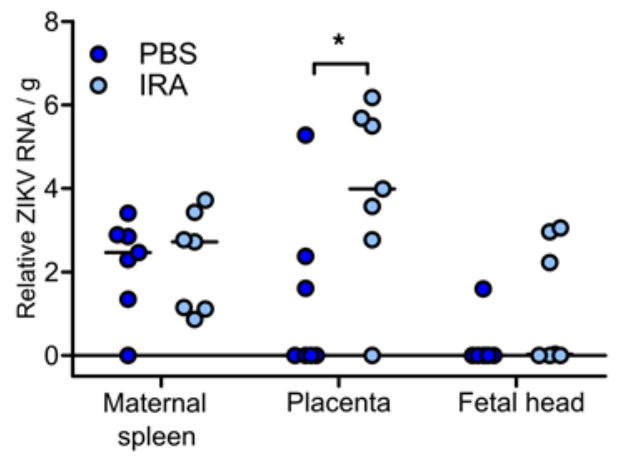

D

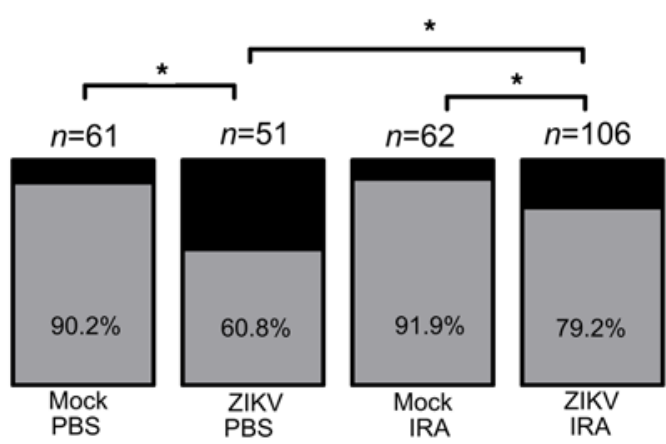

Figure 2. In utero exposure to ZIKV increases placental IL-11. At E10, pregnant CD-1 mice received an intrauterine injection of $10^{6} \mathrm{TCID}_{50}$ units of the 2015 Brazil ZIKV or vehicle (mock). (A) Placentas were collected 6 hours after inoculation ( $6 \mathrm{hpi}$ ), and the mRNA expression of proinflammatory cytokines was quantified (mock: $n=3$; ZIKV: $n=$ 4). (B) The concentration of IL-1 $\beta$ was measured by ELISA from tissue homogenates ( $\mathrm{pg} / 100 \mu \mathrm{g}$ protein). Following ZIKV inoculation, pregnant dams were treated daily with either an IL-1 receptor antagonist (IRA) or PBS until tissue harvesting. (C) ZIKV RNA was isolated and quantified from maternal spleen, placenta, and fetal heads collected 48 hpi. (D) Fetal viability was determined as the percentage of viable fetuses within the inoculated uterine horn. $n=$ total number of fetuses from 8 to 14 dams per group. Student's $t$ test (A and B), and $\chi^{2}$ test (C). ${ }^{*} P<0.05$.

to uninfected dams ( $P<0.05,1$-way ANOVA, Figure 4, B and C). IRA treatment reduced neonatal neuroinflammation as well as placental inflammation; thus, the reduced neuroinflammation in pups might be caused by direct effects of IRA treatment on the CNS or indirect effects on placental inflammation and growth restriction.

In vitro ZIKV infection decreases proliferation of primary microglia, which is reversed with IRA treatment. To explore the direct effects of IRA on ZIKV-induced activation of microglia, primary perinatal 
A

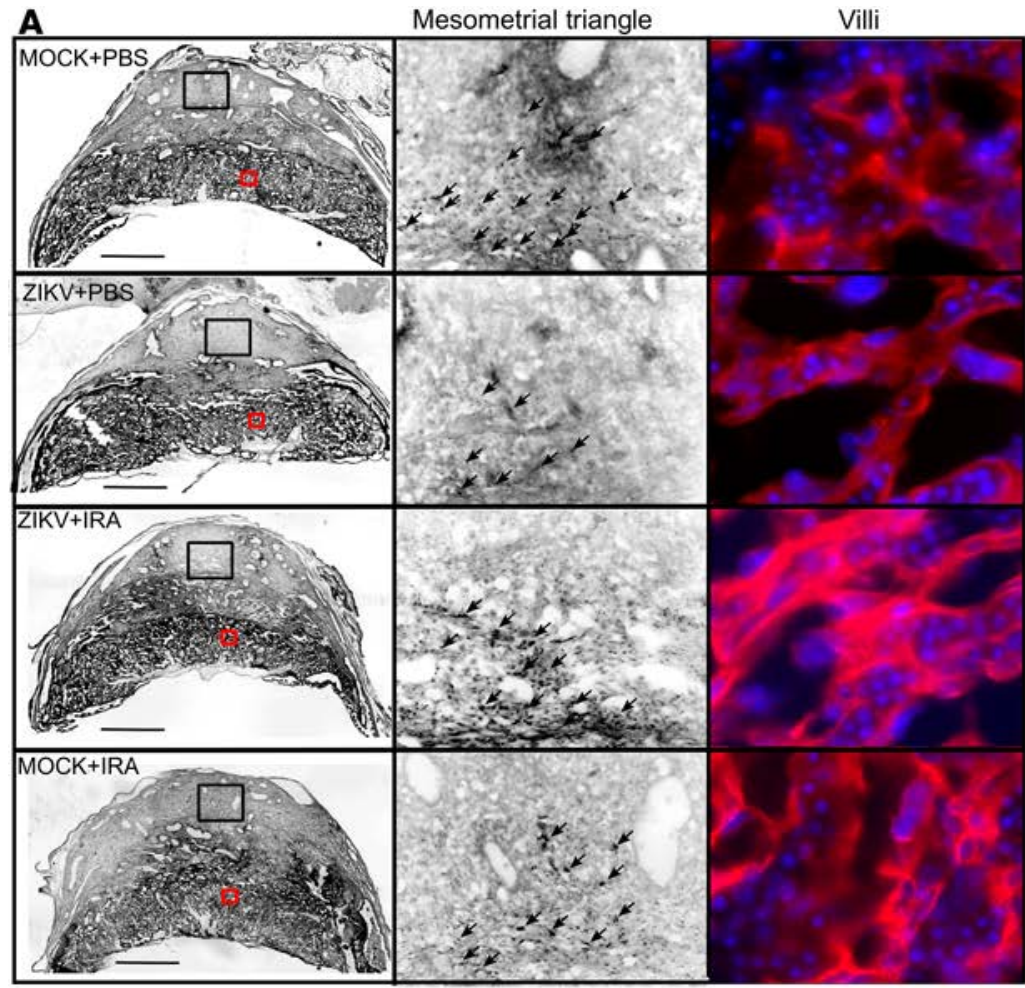

B

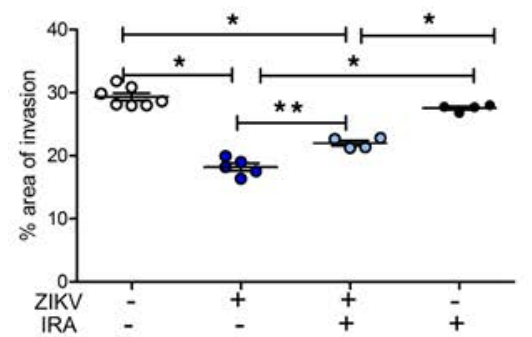

C

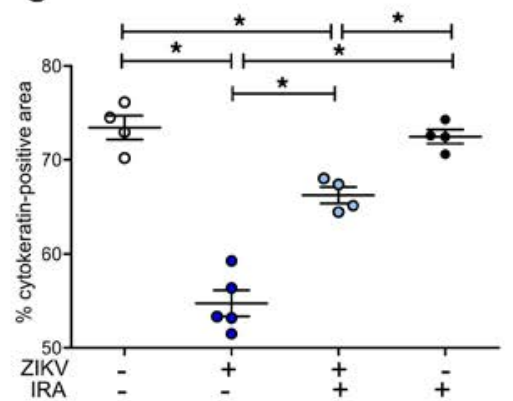

D
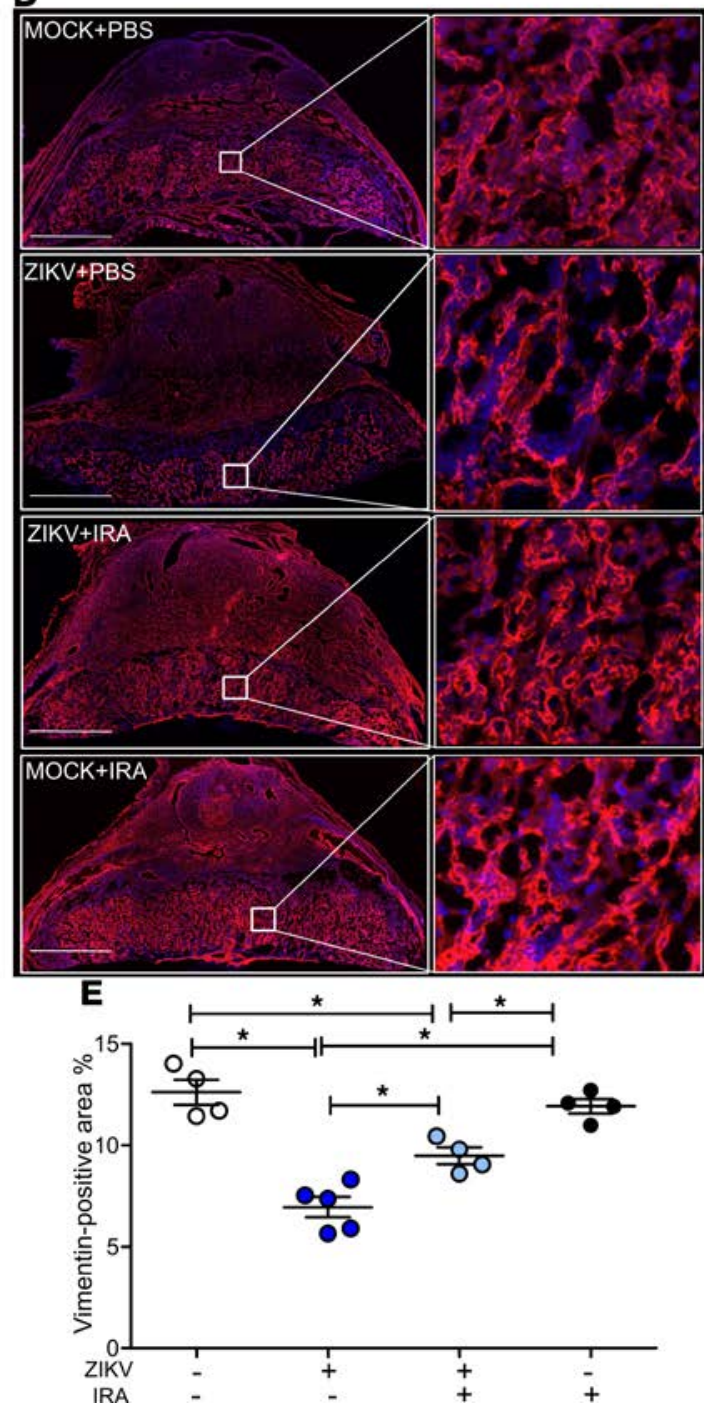

Figure 3. IL-1 receptor antagonism during intrauterine ZIKV infection improves trophoblast invasion and vascular remodeling despite enhanced viral replication. At E10, pregnant CD-1 mice received an intrauterine injection of $10^{6} \mathrm{TCID}_{50}$ units of the 2015 Brazil ZIKV or vehicle (mock). Following ZIKV inoculation, pregnant dams were treated daily with either an IL-1 receptor antagonist (IRA) or PBS until tissue harvesting (E12). (A) Representative fluorescence immunostaining for cytokeratin to label trophoblast cells in the placenta. The middle and right panels are the high magnification of the black boxes (mesometrial triangle; cytokeratin-positive cells are indicated by black arrows) and the red boxes (villi) in the first panels. (B) Quantitative analysis of trophoblast density (as a measure of trophoblast invasion) in the mesometrial triangle (corresponding to the middle panels of $\mathbf{A}$, black arrows). (C) Quantitative analysis of trophoblast density in the villi (corresponding to the right panels of $\mathbf{A}$, red). (D) Representative fluorescence immunostaining for vimentin (red) to label endothelial cells in placenta. (E) Quantitative analysis of endothelial cell density in the villi. One-way ANOVA with Bonferroni's multiple comparisons test (B, C, and $\mathbf{E}){ }^{*} P<0.05$. Scale bar: $1 \mathrm{~mm}$.

microglia were cultured and infected in vitro. Detection of ZIKV protein using IHC demonstrated that more than $90 \%$ of microglia were infected with ZIKV 24 hpi (Figure $5 \mathrm{~A}$ ), and quantification of ZIKV RNA copies revealed no effect of IRA on total viral burden (Figure 5B). To assess the effect of ZIKV infection on microglial proliferation, double IHC staining for microglia (F4/80) and a cell proliferation marker (Ki-67) was performed in cultures $24 \mathrm{hpi}$. Compared with uninfected cultures, ZIKV infection reduced the concentrations of both $\mathrm{F} 4 / 80$ and Ki-67 staining $(P<0.05$, one-way ANOVA, Figure 5, C and D). Treatment of ZIKV-infected cultures with IRA at all concentrations reversed this phenotype and significantly increased concentrations of both F4/80 and Ki-67 staining compared with untreated cultures $(P<0.05,1$-way ANOVA, Figure $5, C$ and D) indicated that IRA treatment can directly reduce neuroinflammation. 


\section{A Negative Geotaxis}

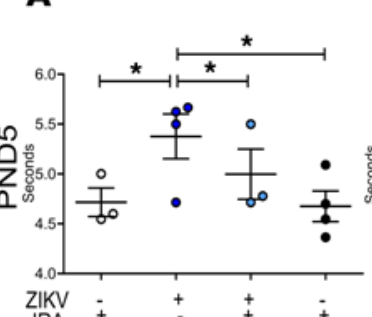

Cliff Aversion
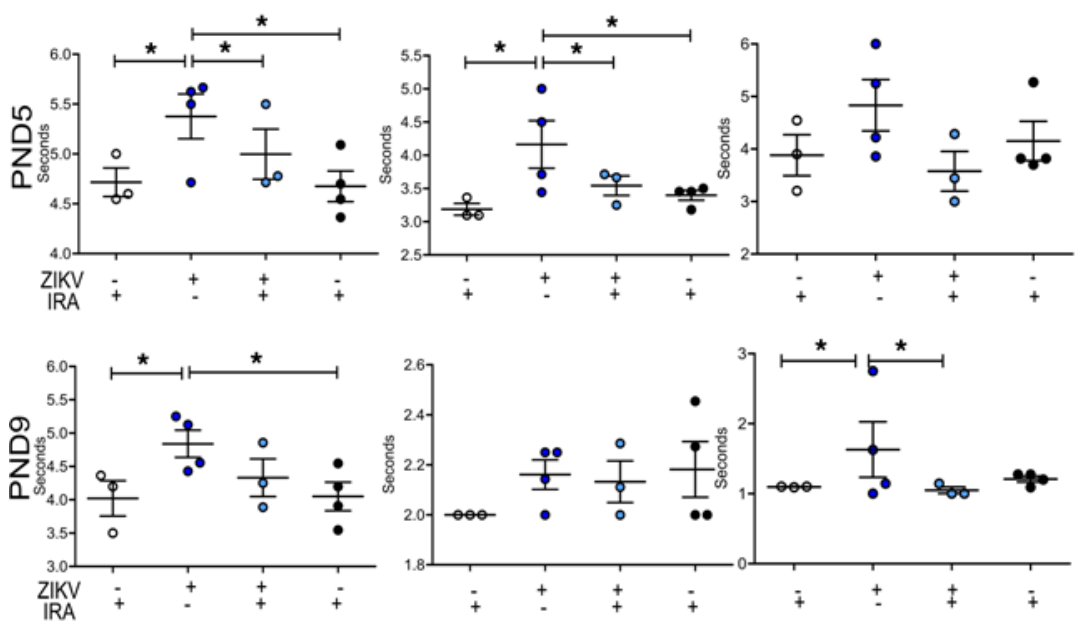

Surface Righting

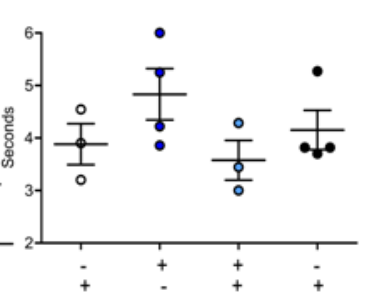

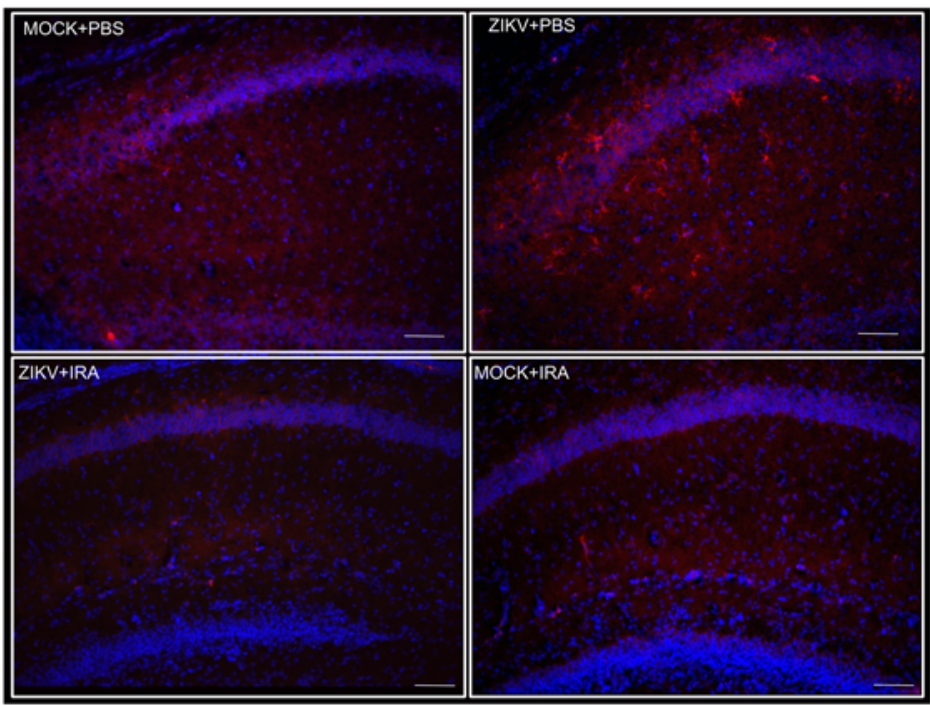

C

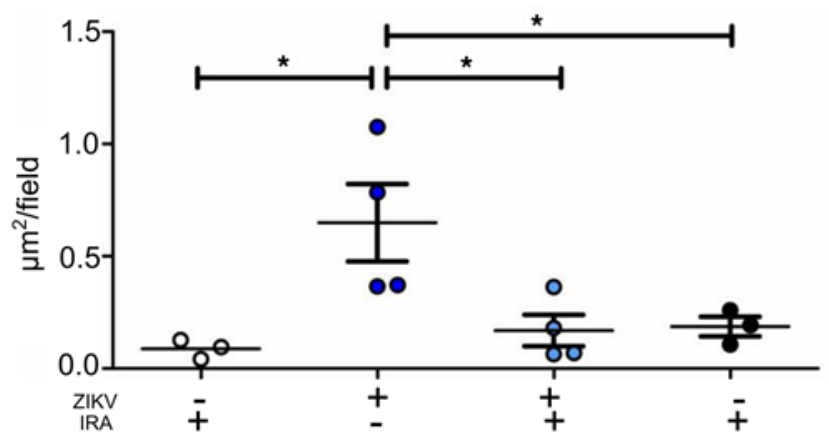

\section{B}

Figure 4. IL-1 receptor antagonism during intrauterine ZIKV infection reverses behavioral abnormalities and neuroinflammation in the pups. At E10, pregnant $C D-1$ mice received an intrauterine injection of $10^{6} \mathrm{TCID}_{50}$ units of the 2015 Brazil ZIKV or vehicle (mock). Following inoculation, pregnant dams were treated daily with either an IL-1 receptor antagonist (IRA) or PBS until E18. (A) Behavioral testing, including negative geotaxis, cliff aversion, and surface righting, was performed on the surviving pups at postnatal day (PND) 5 or 9 to access neurologic development ( $n=3-4$ litters/treatment). Immunohistochemical staining for lba-1 (red) in the brains of pups at PND5. (B) IHC was performed to quantify the concentration of microglia (as a measure of neuroinflammation) in the hippocampus of pups from ZIKVcompared with mock-inoculated dams (C). One-way ANOVA with Bonferroni's multiple comparisons test (C). ${ }^{*} P<0.05$. Scale bars: $50 \mu \mathrm{m}$. 
A
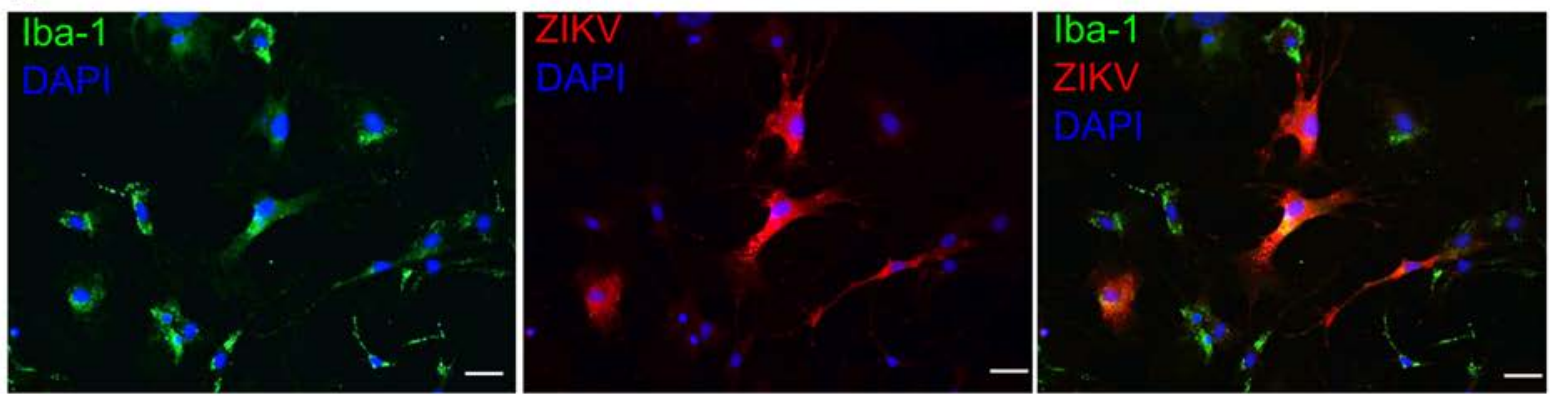

B

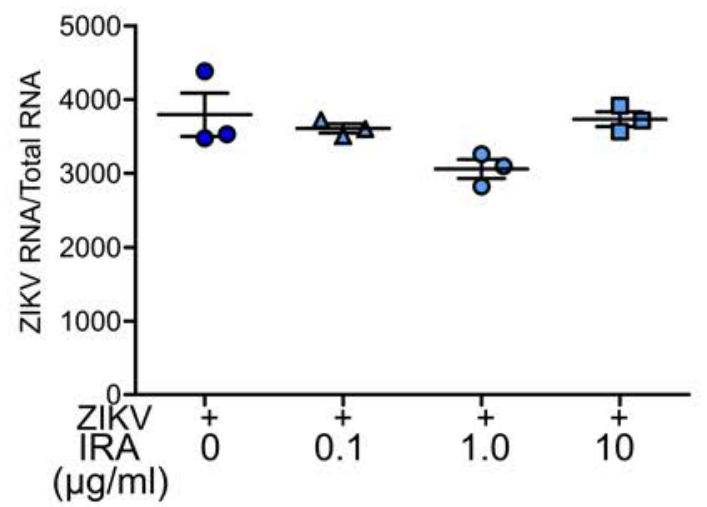

C

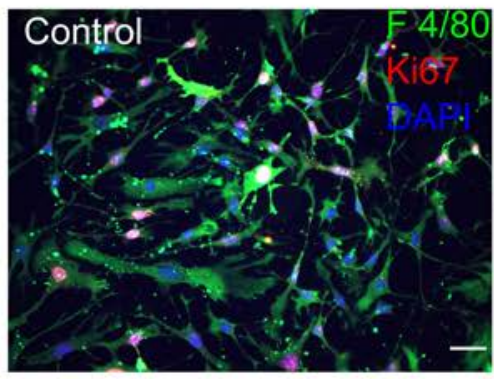

D
F 4/80+

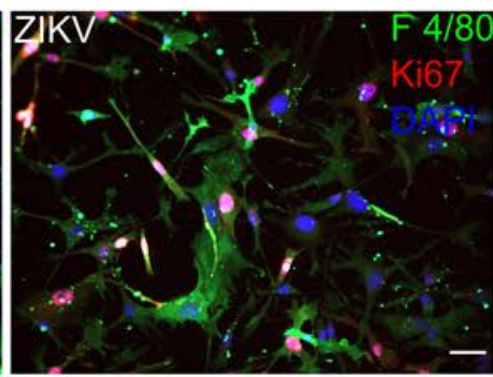

Ki67+

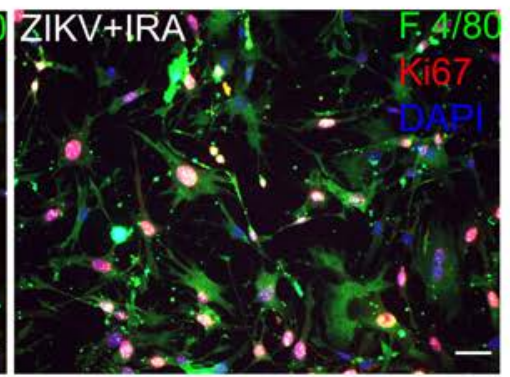

F 4/80+ Ki67+/F 4/80+
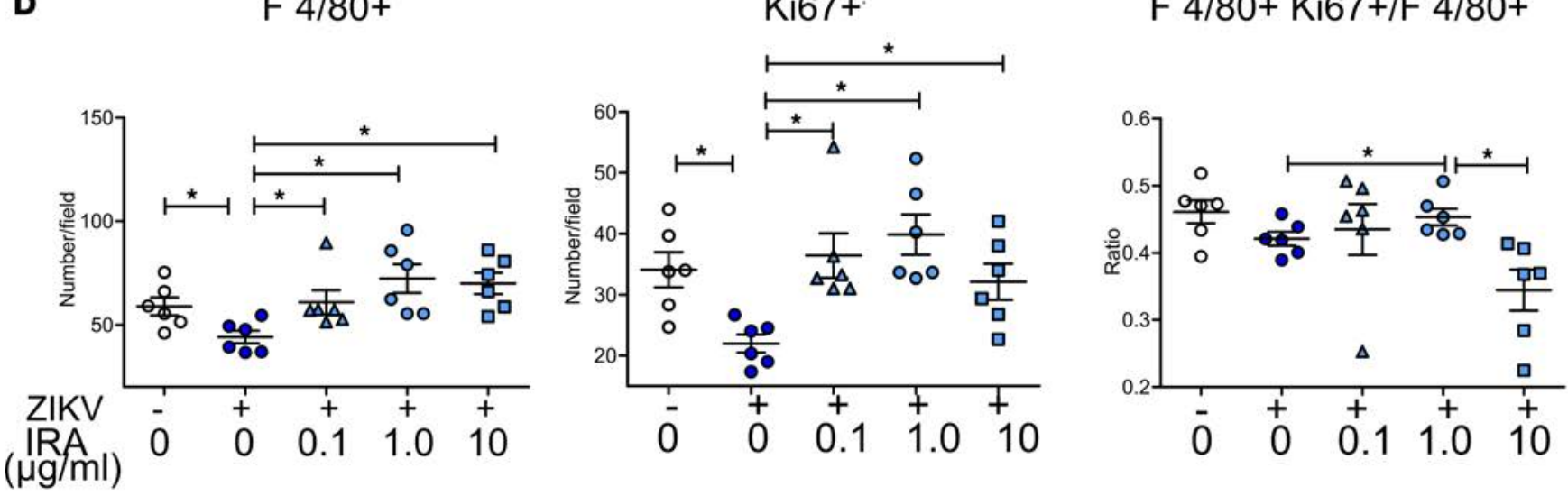

Figure 5. IL-1 receptor antagonist increases microglial proliferation in vitro. Primary microglial cell cultures were generated from brain tissues collected from neonatal mice at P5. Cell cultures were treated with either $5 \times 10^{6}$ PFU of the 2015 Brazil ZIKV or vehicle. One hour after infection (1 hpi), either IRA or PBS was added to the cultures. At 24 hpi, primary cell cultures were either fixed or fresh frozen. (A) Representative fluorescence immunostaining for microglia (Iba-1, green) and ZIKV (red) from ZIKV-infected cultures. (B) Relative ZIKV RNA was quantified by qRT-PCR in microglia. (C) Representative fluorescence immunostaining for F4/80 (microglia, green) and Ki-67 (proliferation marker, red) and quantification of proliferating microglial cells (D). One-way ANOVA with Bonferroni's multiple comparisons test (B and $\mathbf{D})$. All experimental conditions were performed in triplicate, and each experiment was repeated twice. ${ }^{*} P<0.05$. Scale bars: $20 \mu \mathrm{m}$. 
significantly to adverse perinatal outcomes, and should be considered in therapeutic approaches for ZIKV and other infections that cause congenital defects during pregnancy. The maternal inflammatory response represents an environmental factor that is known to increase the risk for childhood and developmental diseases $(51,52)$, such as autism spectrum disorders, schizophrenia, learning disabilities, as well as cognitive and motor deficits (53-55). Although ZIKV infection has direct effects on fetal tissues, we show that placental inflammation is also a major contributor to adverse perinatal outcomes following in utero ZIKV infection.

In our murine model, intrauterine infection with live, replicating ZIKV caused an acute increase in placental concentrations of IL-1 $\beta$, but not of other proinflammatory cytokines, that was associated with fetal demise. Blocking IL-1 $\beta$ signaling improved trophoblast invasion and placental vascularity, fetal viability, and neurobehavioral measures in the offspring. ZIKV is not the only exposure that causes IL-1 $\beta$ mediated inflammation during pregnancy. Previous studies employing intrauterine LPS have established that IL-1 $\beta$-mediated inflammation in the maternal compartment and placenta predicts the extent of fetal neurotoxicity and long-term adverse perinatal sequelae (56-59). Furthermore, IL-1 receptor blockade with IRA decreases activation of neuronal NOS (nNOS), cortical neuronal cell death, and perinatal brain injury, all of which occur with exposure to intrauterine inflammation $(25,60,61)$. Intraperitoneal injections of murine recombinant IL-1 $\beta$ (rIL-1 $\beta$ ) during E15-E17 of gestation result in neurologic sequelae (62), consistent with ZIKV (the present study) and LPS (56, 63-65), illustrating that elevated IL-1 $\beta$ during pregnancy is a significant mediator of adverse perinatal outcomes.

The placenta represents the interface between maternal and fetal circulation, and features an extensive vascular network that facilitates the exchange of oxygen and nutrients, and the removal of waste. Essential to the development of the placenta is sufficient invasion of trophoblasts. Insufficient trophoblast invasion and vascular remodeling are associated with many pregnancy disorders, including preeclampsia and fetal growth restriction (66). In the present study, maternal ZIKV infection significantly delayed the invasion of trophoblasts into the mesometrial triangle of the placenta at a critical time during placental development (i.e., E10.5). Similarly, placental vascular development was dysregulated by in utero ZIKV infection, as evidenced by a significant decrease in endothelial cell density. This disruption to the normal placental architecture may have affected the anchoring of the placenta into the uterine wall and the nutrient exchange between the dam and fetuses, which are necessary for fetal development $(67,68)$.

ZIKV can infect many cell types in the placenta and fetus, including placental trophoblasts and endothelial cells, as well as fetal microglia, endothelial cells, and neural progenitor cells (13, 69, 70). In addition, ZIKV infection causes acute placental inflammation, including secretion of IL-1 $\beta$ (21). Regardless of the cell type that produces IL-1 $\beta$, studies have further shown that inflammatory responses, including IL- $1 \beta$ secretion, are involved in placental dysregulation (i.e., the delay of trophoblast invasion and vascular remodeling) $(59,71,72)$. While maternal treatment with IRA did not reduce ZIKV replication in the placenta, it significantly preserved placental function by increasing trophoblast invasion and improving vascularity, which is necessary for maternal-fetal communication. Furthermore, we found that the placental ZIKV RNA copies did not correlate with fetal survival, suggesting that the presence of ZIKV was not a significant factor in the trophoblast invasion. In contrast, data from the current study illustrate that the virus-induced and IL- $1 \beta$-mediated immune response may be a major factor associated with fetal brain injury, as it allowed more virus to translocate through the inflamed placenta. Thus, ZIKV infection may damage the fetus directly or indirectly through placental dysregulation and inflammation.

The detection of ZIKV in fetal brains of humans (73-77) and mice $(13,78-80)$ has led to the hypothesis that virus replication in the CNS causes adverse perinatal outcomes. Although ZIKV is detectable in the fetal brain $(20,81)$, we have shown that ZIKV is undetectable in the neonatal brain (13). Despite a lack of replicating virus in the neonatal brain, neurologic abnormalities, including altered neurobehavioral outcomes, are observed into adolescence. In the CNS, microglia are a target for ZIKV (13, 82) and express the IL-1 receptor (83). In vitro, IRA treatment improved the proliferation of primary microglia exposed to ZIKV infection. In vivo, at P0 (13) and P5 (the present study), microglial density was increased in the brains of offspring exposed to ZIKV in utero, which was associated with an altered behavioral phenotype. We hypothesize that the continuous activation of microglia after birth may contribute to long-term neurodevelopmental deficits in the offspring (82). Our data support that placental 
inflammation, in particular IL-1 $\beta$ activity, during pregnancy underlies prolonged microglia activation because maternal IRA treatment reduced microglia activation in the brains of ZIKV-exposed offspring; therefore, neurobehavioral abnormalities in pups that were observed following placental inflammation may have been caused either by replicating viruses or nonreplicating inflammatory stimuli.

A significant benefit of our murine model of in utero ZIKV infection is that we are utilizing immunocompetent, outbred mice that mimic the genetic and immunological diversity observed in humans. Most murine models of adverse perinatal outcomes following ZIKV infection involve direct inoculation of neonatal mice $(18,84)$ or infection in type I IFN-deficient dams $(85-88)$. One major drawback of the IFN-deficient mouse models is the lack of critical antiviral innate immune responses, which serve as a first line defense against viruses. Despite having an intact immune system, a limitation with our model is that in an effort to bypass the peripheral immune responses, we inoculate mice into the uterine horn, which is not a natural route of infection but does require virus to traverse the placental barrier. Future studies must examine inflammatory immune responses during pregnancy in humans to confirm that IL-1 $\beta$ is elevated and could serve as a therapeutic target for treatment during ZIKV or other congenital infections.

The well-known and safe clinical profile of IRA, a class B drug in pregnancy, provides opportunities for clinical translation in the treatment of congenital Zika syndrome and other fetal developmental sequelae associated with infections during pregnancy. The steps that need to be taken for these findings to be applied in the clinic are studies evaluating the safety of application during pregnancy, including doses, timing, and long-term effects. These translational studies will have a significant impact on perinatal medicine by enhancing the current understanding of the role of placental inflammation in the pathogenesis of fetal congenital diseases, and by testing the feasibility and implications for in utero immunomodulatory agents. In addition to ZIKV, other neurotrophic flaviviruses, including West Nile virus and Powassan virus, cause adverse placental and fetal outcomes when infection occurs during pregnancy (89). ZIKV may serve as an excellent model for many congenital infections that cause long-term perinatal injury and for which there currently is no cure.

\section{Methods}

ZIKVs. The IB H 30656 (Nigeria, 1968) and PRVABC59 (Puerto Rico, 2015) strains of ZIKV were purchased from ATCC. The Paraíba (Brazil, 2015) ZIKV strain was provided by Stephen Whitehead of the National Institute of Allergy and Infectious Diseases (Bethesda, Maryland, USA). All procedures for handling ZIKV were approved by the Institutional Biosafety Committee. Stocks of ZIKV were generated by infecting Vero cells at an MOI of $0.0150 \%$ tissue culture infectious doses (TCID ${ }_{50}$ ) per cell in DMEM supplemented with $2.5 \% \mathrm{FBS}, 1 \mathrm{mM}$ sodium pyruvate, $100 \mathrm{U} / \mathrm{ml}$ penicillin, $100 \mu \mathrm{g} / \mathrm{ml}$ streptomycin and $2 \mathrm{mM}$ L-glutamine (infection media [IM]). Approximately $72 \mathrm{hpi}$, the infected cell supernatant fluids were collected, clarified by centrifugation at $900 \mathrm{~g}$ for 10 minutes, and stored in aliquots at $-80^{\circ} \mathrm{C}$.

Virus inactivation was achieved by incubating the infected cell supernatant with $\beta$-propiolactone (BPA; final concentration $0.05 \% \mathrm{v} / \mathrm{v}$ ) at $4^{\circ} \mathrm{C}$ for 24 hours. The supernatant was then incubated at $37^{\circ} \mathrm{C}$ for 2 hours to inactivate the BPA (90). No infectivity was detected by TCID $_{50}$ assay after BPA treatment, verifying complete virus inactivation.

Mouse experiments. Timed-pregnant adult (2-3 months of age) CD-1 mice were purchased from Charles River Laboratories. Animals arrived at E9 and were housed in a specific pathogen-free facility at Johns Hopkins University with ad libitum access to food and water. Dams were allowed to acclimate for more than 24 hours, which is sufficient time for most basic measures of stress and immune function to return to baseline in CD-1 mice (91). All experimental procedures were performed at the same time of day.

At E10, mice were anesthetized continuously with isoflurane and underwent mini-laparotomy in the lower abdomen for ZIKV injections. Animals were randomly assigned to receive either $10^{6} \mathrm{TCID}_{50}$ units of ZIKV or inactivated ZIKV suspended in $100 \mu 1$ DMEM or $100 \mu 1$ DMEM alone as previously described (13). For intrauterine injection, the inoculum was divided equally into 4 injections delivered into the uterine myometrium, opposite the placenta, and between the gestational sacs of the first 5 fetuses closest to the cervix of one uterine horn. The contralateral uterine horn was not manipulated. Routine closure was performed after injections, and dams were returned to individual cages for recovery. Investigators were not blinded to the treatment group assignments. Mice were randomly selected to be euthanized by $\mathrm{CO}_{2}$ exposure followed by cardiac exsanguination at either 6 or 48 hpi, or after delivery at P5, P9, or P18, depending on the experimental design. At the time of euthanasia, the total number of viable 
and nonviable fetuses was quantified for each pregnant dam. Fetal viability was determined as the percentage of fetuses within the inoculated uterine horn for ZIKV- and mock-infected dams that were viable. Small or discolored fetuses or the absence of a fetus at an implantation site were counted as nonviable. Maternal spleens, placentas, and fetuses were collected from each dam for determination of viral burden by quantitative RT-PCR (qRT-PCR), TCID ${ }_{50}$ assay, and IHC. All animal studies were conducted under animal biosafety level 2 (BSL2) containment.

IRA treatment. Beginning 1, 3, or 24 hpi, dams were injected intraperitoneally with either IRA (Kineret, or anakinra, Amgen) at $10 \mathrm{mg} / \mathrm{kg}$ in $100 \mu \mathrm{PBS}$ (24) or with the same volume of PBS (vehicle control). The injections were repeated every 24 hours after the first treatment until tissue harvest (half-life, 4-6 hours; ref. 92). The dose and route were selected based on our previous study (24) and another study (23) demonstrating that at this dose, the antagonist effectively protects against the placental and neurodevelopmental deficits in animal models of maternal inflammation.

ELISA. According to the manufacturer's protocol, an ELISA (Abcam) was performed to measure the IL-1 $\beta$ content in fresh frozen placentas and maternal spleens.

$q R T-P C R$. Tissue homogenates were stored at $-80^{\circ} \mathrm{C}$. Total RNA was extracted from tissue samples using the RNeasy Lipid Tissue Mini Kit (QIAGEN), according to the manufacturer's instructions. ZIKV RNA levels were determined by one-step qRT-PCR reaction using the QuantiTect Probe RT-PCR Kit (QIAGEN) according to the manufacturer's protocol. The real-time PCR primers and probe for ZIKV RNA detection were the ZIKV 1162c set described previously (93), and the primer sequences and concentrations were as follows: Fwd $(100 \mu \mathrm{M}), 5^{\prime}$-CCGCTGCCCAACACAAG-3'; Rev $(100 \mu \mathrm{M})$, 5'-CCACTAACGTTCTTTTGCAGACAT-3'; probe $(25 \mu \mathrm{M})$, 5'-/56-FAM/AGCCTACCT/ZEN/TGACAAGCAATCAGACACTCAA/3IABkFQ/-3' (Integrated DNA Technologies). ZIKV RNA copies were determined relative to a standard curve produced using serial 10-fold dilutions of ZIKV RNA isolated from ZIKV stocks with a known infectious virus titer.

PCR multiplex array. Placentas were collected at 6 and 48 hpi and fresh frozen on dry ice, followed by long-term storage at $-80^{\circ} \mathrm{C}$. For each placenta, $2 \mu \mathrm{g}$ RNA was used for complementary cDNA synthesis in a 40- $\mu 1$ reaction, using Bio-Rad iScript cDNA Synthesis Kit (no. 170-8891, Bio-Rad). A TaqMan Mouse Immune Array v2.1 (no. 4365297, Thermo Fisher Scientific) was used to evaluate 96 immune markers. To carry out this array, $100 \mu 1$ of $2 \times$ iTaq Super Mix (Bio-Rad, no. 172-5134) was diluted with $60 \mu 1$ of water and the $40 \mu 1$ of the prepared cDNA. Array analyses were performed with the Quant Studio 12K Flex Real-Time PCR System (Thermo Fisher Scientific) for 40 cycles. The Thermo Fisher Connect cloud system was used for data storage.

Primary microglia cultures. Neonatal brains were isolated from pups at P5, and the tissue was dissociated into the single-cell suspension using a Neural Tissue Dissociation Kit (Miltenyi Biotec). Microglia were then isolated from the single-cell suspension using CD11b (a microglial marker) MicroBeads (Miltenyi Biotec) according to the manufacturer's instructions, and plated at a density of $1 \times 10^{4}$ cell/ $\mathrm{cm}^{2}$. Microglial cultures were infected with either ZIKV (Brazil strain, $5 \times 10^{6}$ ) or media with or without IRA. After 24 hours, cells were fresh frozen or fixed in 4\% paraformaldehyde (PFA) for 20 minutes and stored in PBS. All experimental conditions were performed in triplicate, and each experiment was repeated 3 times for RNA extraction and 6 times for immunofluorescence imaging.

IHC and immunofluorescence imaging. At E12, pregnant mice were euthanized with carbon dioxide, and their embryos were harvested. Isolated whole brains and placentas were fixed overnight at $4{ }^{\circ} \mathrm{C}$ in $4 \% \mathrm{PFA}$. The following day, the brains and placentas were washed extensively with PBS and immersed in $30 \%$ sucrose until saturation. Using a Leica CM1950 cryostat, the specimens were cut at 20- $\mu$ m thickness and mounted on positively charged slides (Fischer Scientific), followed by IHC. Slides were washed with PBS, which was followed by incubation in PBS solution containing 0.05\% Triton X-100 and 5\% normal goat serum (Invitrogen) for 30 minutes. Placentas were incubated with rabbit anti-vimentin (1:200, ab92547, clone EPR3776, Abcam)/cytokeratin antibodies (1:200, Z0622, Dako) overnight at $4^{\circ} \mathrm{C}$. Fetal brains were incubated with rabbit anti-Iba-1 antibody (1:200, 019-1974, Wako). The in vitro microglia were incubated with mouse antiZIKV (1:100, MAB10216, clone D1-4G2-4-15, EMD Millipore), rabbit anti-Iba-1/rat anti-F4/80 (1:400, NB600-404, clone CI-A3-1, Novus) and rabbit anti-Ki-67 (1:200, ab15580, Abcam). The next day, sections were rinsed with PBS and then incubated with donkey anti-rabbit (A10042)/mouse (A10037) Alexa Fluor 568, or donkey anti-rabbit (A21206)/rat (A21208) Alexa Fluor 488 (Life Technologies) fluorescent secondary antibody diluted in 1:500 for 3 hours at room temperature. DAPI (10236276001, Roche) was applied for counterstaining, followed by mounting with Fluoromount-G (eBioscience). 
Behavioral quantification. Neurobehavioral testing was performed on pups at P5 or P9 using a modified developmental milestone scoring system, as previously described (94). Briefly, motor development was assessed by negative geotaxis and surface righting, and cognitive development was measured by cliff aversion. Negative geotaxis is to test the innate postural response of pups to rotate $180^{\circ}$ from the head-down position to the head-up position on an inclined plane. Surface righting is to test the reflex that corrects the orientation of the body when pups are taken out of its normal upright position. The cliff aversion test prompts pups to change position, escaping away from the edge.

Statistics. All data were analyzed with GraphPad Prism software. Fetal viability data were analyzed with a $\chi^{2}$ test. ZIKV RNA data were analyzed using multiple $t$ tests or 1-way ANOVAs with Bonferroni's post hoc corrections for multiple comparisons. Correlations were analyzed using Spearman's correlation test. Cytokine data were analyzed with Student's $t$ test. Quantifications of IHC (e.g., trophoblast invasion, vascular density, microglia concentration), and behavioral testing data were analyzed using 1-way ANOVAs with Bonferroni's post hoc corrections for multiple comparisons. Data are presented as mean \pm SEM. Mean differences were considered statistically significant at $P<0.05$.

Study approval. This study was carried out in accordance with the recommendations in the Guide for the Care and Use of Laboratory Animals (National Academies Press, 2011). The protocols were approved by the Institutional Animal Care and Use Committee and Health Safety and the Environment Committee of Johns Hopkins University.

\section{Author contributions}

IB, SLK, and JL conceptualized the experiments; IB, JL, MSV, AB, AP, and SLK designed the experiments; JL, MSV, BJ, HX, LX, MWM, AB, AP, and IB conducted the experiments; JL, MSV, IB, and $\mathrm{AB}$ analyzed data; JL and MSV graphed data; IB, SLK, JL, AP, and MSV wrote the manuscript; SLK, IB, JL, MSV, AB, and JSS edited the manuscript; all authors approved the final draft of the manuscript.

\section{Acknowledgments}

The authors thank the expert animal care staff at Johns Hopkins for assistance with maintenance of ZIKV-infected dams in our facilities. This work was supported by the Integrated Research Center for Fetal Medicine (IB), Sheikh Abdullah Bugshan Fund (IB), Sherrilyn and Ken Fisher Center for Environmental Infectious Diseases (SLK), ABOG/AAOGF Bridge Fund (IB), NIH R01HD097608 (IB and SK), and NIH T32 OD011089 Training Veterinarians for Careers in Biomedical Research (MSV).

Address correspondence to: Irina Burd, Integrated Research Center for Fetal Medicine and Department of Gynecology and Obstetrics, Johns Hopkins University School of Medicine, 600 North Wolfe Street, Phipps 217, Baltimore, Maryland 21287, USA. Phone: 410.955.8496. Email: Iburd@jhmi.edu. Or to: Sabra L. Klein, W. Harry Feinstone Department of Molecular Microbiology and Immunology, Johns Hopkins Bloomberg School of Public Health, 615 North Wolfe Street, Room W2118, Baltimore, Maryland 21205, USA. Phone: 410.955.8898; Email: Sklein2@jhu.edu.

1. WHO. Situation Report: Zika Virus, Microcephaly, Guillain-Barré Syndrome. https://www.who.int/emergencies/zika-virus/situation-report/10-march-2017/en/. Posted March 10, 2017. Accessed February 28, 2019.

2. Pan American Health Organization/WHO. Zika Cases and Congenital Syndrome Associated with Zika Virus Reported by Countries and Territories in the Americas, 2015-2017 Cumulative Cases. https://www.paho.org/hq/dmdocuments/2017/2017-ago-10-phe-ZIKV-casess.pdf. Updated August 10, 2017. Accessed February 25, 2019.

3. Mead PS, Hills SL, Brooks JT. Zika virus as a sexually transmitted pathogen. Curr Opin Infect Dis. 2018;31(1):39-44.

4. Bagasra O, Addanki KC, Goodwin GR, Hughes BW, Pandey P, McLean E. Cellular targets and receptor of sexual transmission of zika virus. Appl Immunohistochem Mol Morphol. 2017;25(10):679-686.

5. Bierlaire D, Mauguin S, Broult J, Musso D. Zika virus and blood transfusion: the experience of French Polynesia. Transfusion. 2017;57(3 pt 2):729-733.

6. Miner JJ, Diamond MS. Zika virus pathogenesis and tissue tropism. Cell Host Microbe. 2017;21(2):134-142.

7. Wahid B, Ali A, Rafique S, Idrees M. Current status of therapeutic and vaccine approaches against Zika virus. Eur J Intern Med. 2017;44:12-18.

8. Sirohi D, Kuhn RJ. Can an FDA-approved Alzheimer's drug be repurposed for alleviating neuronal symptoms of Zika virus? MBio. 2017;8(3):e00916-17.

9. Stein DR, et al. Human polyclonal antibodies produced in transchromosomal cattle prevent lethal Zika virus infection and testicular atrophy in mice. Antiviral Res. 2017;146:164-173.

10. Magnani DM, et al. Fetal demise and failed antibody therapy during Zika virus infection of pregnant macaques. Nat Com- 
mun. 2018;9(1):1624.

11. Mor G. Placental inflammatory response to Zika virus may affect fetal brain development. Am J Reprod Immunol. 2016;75(4):421-422

12. Quicke KM, et al. Zika virus infects human placental macrophages. Cell Host Microbe. 2016;20(1):83-90.

13. Vermillion MS, et al. Intrauterine Zika virus infection of pregnant immunocompetent mice models transplacental transmission and adverse perinatal outcomes. Nat Commun. 2017;8:14575.

14. Olmo IG, et al. Zika virus promotes neuronal cell death in a non-cell autonomous manner by triggering the release of neurotoxic factors. Front Immunol. 2017;8:1016

15. Alcendor DJ. Zika virus infection of the human glomerular cells: implications for viral reservoirs and renal pathogenesis. $J$ Infect Dis. 2017;216(2):162-171.

16. Lum FM, et al. Zika virus infects human fetal brain microglia and induces inflammation. Clin Infect Dis. 2017;64(7):914-920.

17. Tricarico PM, Caracciolo I, Crovella S, D'Agaro P. Zika virus induces inflammasome activation in the glial cell line U87MG. Biochem Biophys Res Commun. 2017;492(4):597-602.

18. Manangeeswaran M, Ireland DD, Verthelyi D. Zika (PRVABC59) infection is associated with T cell infiltration and neurodegeneration in CNS of immunocompetent neonatal C57B1/6 mice. PLoS Pathog. 2016;12(11):e1006004.

19. Khaiboullina SF, Uppal T, Sarkar R, Gorzalski A, St Jeor S, Verma SC. ZIKV infection regulates inflammasomes pathway for replication in monocytes. Sci Rep. 2017;7(1):16050.

20. Shao Q, et al. Zika virus infection disrupts neurovascular development and results in postnatal microcephaly with brain damage. Development. 2016;143(22):4127-4136.

21. Tappe D, et al. Cytokine kinetics of Zika virus-infected patients from acute to reconvalescent phase. Med Microbiol Immunol. 2016;205(3):269-273.

22. Pillay V, Savage N, Laburn H. Interleukin-1 receptor antagonist in newborn babies and pregnant women. Pflugers Arch. 1993;424(5-6):549-551.

23. Girard S, Tremblay L, Lepage M, Sébire G. IL-1 receptor antagonist protects against placental and neurodevelopmental defects induced by maternal inflammation. J Immunol. 2010;184(7):3997-4005.

24. Leitner K, Al Shammary M, McLane M, Johnston MV, Elovitz MA, Burd I. IL-1 receptor blockade prevents fetal cortical brain injury but not preterm birth in a mouse model of inflammation-induced preterm birth and perinatal brain injury. Am $J$ Reprod Immunol. 2014;71(5):418-426.

25. Rosenzweig JM, Lei J, Burd I. Interleukin-1 receptor blockade in perinatal brain injury. Front Pediatr. 2014;2:108.

26. Gorman MJ, et al. An immunocompetent mouse model of Zika virus infection. Cell Host Microbe. 2018;23(5):672-685.e6.

27. Aliota MT, Caine EA, Walker EC, Larkin KE, Camacho E, Osorio JE. Characterization of lethal zika virus infection in AG129 mice. PLoS Negl Trop Dis. 2016;10(4):e0004682.

28. Lazear HM, Govero J, Smith AM, et al. A mouse model of Zika virus pathogenesis. Cell Host Microbe. 2016;19(5):720-730

29. Bowen JR, et al. Zika virus antagonizes type I interferon responses during infection of human dendritic cells. PLoS Pathog. 2017;13(2):e1006164.

30. Kumar A, et al. Zika virus inhibits type-I interferon production and downstream signaling. EMBO Rep. 2016;17(12):1766-1775

31. Tripathi S, et al. A novel Zika virus mouse model reveals strain specific differences in virus pathogenesis and host inflammatory immune responses. PLoS Pathog. 2017;13(3):e1006258.

32. Rossi SL, et al. Characterization of a novel murine model to study Zika virus. Am J Trop Med Hyg. 2016;94(6):1362-1369.

33. Dowall SD, et al. A susceptible mouse model for Zika virus infection. PLoS Negl Trop Dis. 2016;10(5):e0004658.

34. Elong Ngono A, et al. Mapping and role of the CD8+ T cell response during primary zika virus infection in mice. Cell Host Microbe. 2017;21(1):35-46.

35. Tang WW, Young MP, Mamidi A, Regla-Nava JA, Kim K, Shresta S. A mouse model of Zika virus sexual transmission and vaginal viral replication. Cell Rep. 2016;17(12):3091-3098

36. Yockey LJ, et al. Vaginal exposure to Zika virus during pregnancy leads to fetal brain infection. Cell. 2016;166(5):1247-1256.e4

37. Sones JL, Davisson RL. Preeclampsia, of mice and women. Physiol Genomics. 2016;48(8):565-572.

38. Wang W, Li G, De W, et al. Zika virus infection induces host inflammatory responses by facilitating NLRP3 inflammasome assembly and interleukin-1beta secretion. Nat Commun. 2018;9(1):106.

39. Cui Y, et al. Role of corin in trophoblast invasion and uterine spiral artery remodelling in pregnancy. Nature. 2012;484(7393):246-250.

40. Geusens N, et al. Changes in endovascular trophoblast invasion and spiral artery remodelling at term in a transgenic preeclamptic rat model. Placenta. 2010;31(4):320-326.

41. Dada T, et al. Mouse model of intrauterine inflammation: sex-specific differences in long-term neurologic and immune sequelae. Brain Behav Immun. 2014;38:142-150.

42. Novak CM, et al. Progesterone improves perinatal neuromotor outcomes in a mouse model of intrauterine inflammation via immunomodulation of the placenta. Am J Reprod Immunol. 2018;79(5):e12842.

43. Lei J, et al. Maternal glucose supplementation in a murine model of chorioamnionitis alleviates dysregulation of autophagy in fetal brain. Reprod Sci. 2018;25(8):1175-1185.

44. Lei J, et al. Maternal CD8+ T-cell depletion alleviates intrauterine inflammation-induced perinatal brain injury. Am J Reprod Immunol. 2018;79(5):e12798.

45. Basile K, Kok J, Dwyer DE. Zika virus: what, where from and where to? Pathology. 2017;49(7):698-706.

46. McKenney SL, Mansouri FF, Everett AD, Graham EM, Burd I, Sekar P. Glial fibrillary acidic protein as a biomarker for brain injury in neonatal CHD. Cardiol Young. 2016;26(7):1282-1289.

47. Attaway DF, Waters NM, Geraghty EM, Jacobsen KH. Zika virus: endemic and epidemic ranges of Aedes mosquito transmission. J Infect Public Health. 2017;10(1):120-123.

48. Muthumani $\mathrm{K}$, et al. In vivo protection against ZIKV infection and pathogenesis through passive antibody transfer and active immunisation with a prMEnv DNA vaccine. NPJ Vaccines. 2016;1:16021.

49. Alam A, Ali S, Ahamad S, Malik MZ, Ishrat R. From ZikV genome to vaccine: in silico approach for the epitope-based pep- 
tide vaccine against Zika virus envelope glycoprotein. Immunology. 2016;149(4):386-399.

50. Mumtaz N, van Kampen JJ, Reusken CB, Boucher CA, Koopmans MP. Zika virus: where is the treatment? Curr Treat Options Infect Dis. 2016;8:208-211.

51. Migale R, et al. Specific lipopolysaccharide serotypes induce differential maternal and neonatal inflammatory responses in a murine model of preterm labor. Am J Pathol. 2015;185(9):2390-2401.

52. Stigger $\mathrm{F}$, et al. Inflammatory response and oxidative stress in developing rat brain and its consequences on motor behavior following maternal administration of LPS and perinatal anoxia. Int J Dev Neurosci. 2013;31(8):820-827.

53. Chow $\mathrm{KH}$, Yan Z, Wu WL. Induction of maternal immune activation in mice at mid-539 gestation stage with viral mimic poly(I:C). J Vis Exp. 3643;2016(109):e53643.

54. Elovitz MA, Brown AG, Breen K, Anton L, Maubert M, Burd I. Intrauterine inflammation, insufficient to induce parturition, still evokes fetal and neonatal brain injury. Int J Dev Neurosci. 2011;29(6):663-671.

55. Kirsten TB, Lippi LL, Bevilacqua E, Bernardi MM. LPS exposure increases maternal corticosterone levels, causes placental injury and increases IL-1 $\beta$ levels in adult rat offspring: relevance to autism. PLoS ONE. 2013;8(12):e82244.

56. Gayle DA, Beloosesky R, Desai M, Amidi F, Nuñez SE, Ross MG. Maternal LPS induces cytokines in the amniotic fluid and corticotropin releasing hormone in the fetal rat brain. Am J Physiol Regul Integr Comp Physiol. 2004;286(6):R1024-R1029.

57. Scott LM, Bryant AH, Rees A, Down B, Jones RH, Thornton CA. Production and regulation of interleukin-1 family cytokines at the materno-fetal interface. Cytokine. 2017;99:194-202.

58. Nadeau-Vallée M, et al. Antenatal suppression of IL-1 protects against inflammation-induced fetal injury and improves neonatal and developmental outcomes in mice. J Immunol. 2017;198(5):2047-2062.

59. Li L, Tu J, Jiang Y, Zhou J, Yabe S, Schust DJ. Effects of lipopolysaccharide on human first trimester villous cytotrophoblast cell function in vitro. Biol Reprod. 2016;94(2):33.

60. Savard A, Brochu ME, Chevin M, Guiraut C, Grbic D, Sébire G. Neuronal self-injury mediated by IL-1 $\beta$ and MMP-9 in a cerebral palsy model of severe neonatal encephalopathy induced by immune activation plus hypoxia-ischemia. J Neuroinflammation. 2015;12:111.

61. Tsimis ME, et al. P2X7 receptor blockade prevents preterm birth and perinatal brain injury in a mouse model of intrauterine inflammation. Biol Reprod. 2017;97(2):230-239.

62. Parmentier JH, et al. Regulation of CYP4A1 and peroxisome proliferator-activated receptor alpha expression by interleukin-1beta, interleukin-6, and dexamethasone in cultured fetal rat hepatocytes. Biochem Pharmacol. 1997;54(8):889-898.

63. Dayton ET, Major EO. Recombinant human interleukin 1 beta induces production of prostaglandins in primary human fetal astrocytes and immortalized human fetal astrocyte cultures. J Neuroimmunol. 1996;71(1-2):11-18.

64. Beloosesky R, et al. Prophylactic maternal n-acetylcysteine before lipopolysaccharide suppresses fetal inflammatory cytokine responses. Am J Obstet Gynecol. 2009;200(6):665.e1-665.e5.

65. Kumral A, et al. Erythropoietin attenuates lipopolysaccharide-induced white matter injury in the neonatal rat brain. Neonatology. 2007;92(4):269-278.

66. O’Tierney-Ginn P, Presley L, Minium J, Hauguel deMouzon S, Catalano PM. Sex-specific effects of maternal anthropometrics on body composition at birth. Am J Obstet Gynecol. 2014;211(3):292.e1-292.e9.

67. Lamhot VB, et al. Magnesium sulfate prevents maternal inflammation-induced impairment of learning ability and memory in rat offspring. Am J Obstet Gynecol. 2015;213(6):851.e1-851.e8.

68. Szaba FM, et al. Zika virus infection in immunocompetent pregnant mice causes fetal damage and placental pathology in the absence of fetal infection. PLoS Pathog. 2018;14(4):e1006994.

69. Aagaard KM, et al. Primary Human placental trophoblasts are permissive for Zika virus (ZIKV) replication. Sci Rep. 2017;7:41389.

70. Tabata T, et al. Zika virus targets different primary human placental cells, suggesting two routes for vertical transmission. Cell Host Microbe. 2016;20(2):155-166.

71. Huang SJ, Chen CP, Schatz F, Rahman M, Abrahams VM, Lockwood CJ. Pre-eclampsia is associated with dendritic cell recruitment into the uterine decidua. J Pathol. 2008;214(3):328-336.

72. Lockwood CJ, et al. Decidual hemostasis, inflammation, and angiogenesis in pre-eclampsia. Semin Thromb Hemost. 2011;37(2):158-164.

73. Lin MY, et al. Zika virus infects intermediate progenitor cells and post-mitotic committed neurons in human fetal brain tissues. Sci Rep. 2017;7(1):14883.

74. Mlakar J, Korva M, Tul N, et al. Zika virus associated with microcephaly. N Engl J Med. 2016;374(10):951-958.

75. Tang H, et al. Zika Virus infects human cortical neural progenitors and attenuates their growth. Cell Stem Cell. 2016;18(5):587-590.

76. Garcez PP, et al. Zika virus impairs growth in human neurospheres and brain organoids. Science. 2016;352(6287):816-818

77. McGrath EL, et al. Differential responses of human fetal brain neural stem cells to zika virus infection. Stem Cell Reports. 2017;8(3):715-727.

78. Zhang F, et al. American strain of zika virus causes more severe microcephaly than an old Asian strain in neonatal mice. EBioMedicine. 2017;25:95-105.

79. Wu KY, et al. Vertical transmission of Zika virus targeting the radial glial cells affects cortex development of offspring mice. Cell Res. 2016;26(6):645-654.

80. Cugola FR, et al. The Brazilian Zika virus strain causes birth defects in experimental models. Nature. 2016;534(7606):267-271.

81. Li C, Xu D, Ye Q, et al. Zika virus disrupts neural progenitor development and leads to microcephaly in mice. Cell Stem Cell. 2016;19(5):672.

82. Wang J, et al. Zika virus infected primary microglia impairs NPCs proliferation and differentiation. Biochem Biophys Res Commun. 2018;497(2):619-625.

83. Liu JS, Amaral TD, Brosnan CF, Lee SC. IFNs are critical regulators of IL-1 receptor antagonist and IL-1 expression in human microglia. J Immunol. 1998;161(4):1989-1996.

84. Fernandes NC, et al. Experimental Zika virus infection induces spinal cord injury and encephalitis in newborn Swiss mice. 
Exp Toxicol Pathol. 2017;69(2):63-71.

85. Aliota MT, et al. Zika in the Americas, year 2: what have we learned? what gaps remain? A report from the Global Virus Network. Antiviral Res. 2017;144:223-246.

86. Aliota MT, et al. Heterologous protection against Asian Zika virus challenge in rhesus macaques. PLoS Negl Trop Dis. 2016;10(12):e0005168

87. Kaid C, et al. Zika virus selectively kills aggressive human embryonal CNS tumor cells in vitro and in vivo. Cancer Res. 2018;78(12):3363-3374.

88. Miner JJ, et al. Zika virus infection during pregnancy in mice causes placental damage and fetal demise. Cell. 2016;165(5):1081-1091.

89. Platt DJ, Smith AM, Arora N, Diamond MS, Coyne CB, Miner JJ. Zika virus-related neurotropic flaviviruses infect human placental explants and cause fetal demise in mice. Sci Transl Med. 2018;10(426):eaao7090.

90. Schaecher SR, Mackenzie JM, Pekosz A. The ORF7b protein of severe acute respiratory syndrome coronavirus (SARS-CoV) is expressed in virus-infected cells and incorporated into SARS-CoV particles. $J$ Virol. 2007;81(2):718-731.

91. Fuentealba LC, Rompani SB, Parraguez JI, et al. Embryonic origin of postnatal neural stem cells. Cell. 2015;161(7):1644-1655

92. Akash MS, Rehman K, Chen S. IL-1Ra and its delivery strategies: inserting the association in perspective. Pharm Res. 2013;30(11):2951-2966.

93. Lanciotti RS, et al. Genetic and serologic properties of Zika virus associated with an epidemic, Yap State, Micronesia, 2007. Emerging Infect Dis. 2008;14(8):1232-1239.

94. Gozes I, ed. Neuropeptide Techniques. Totawa, New Jersey, USA: Human Press; 2007. 